\title{
Corela
}

Cognition, représentation, langage

HS-35 | 2022

Anaphore et pronoms en anglais : convergences, différences et complémentarité de quelques approches linguistiques

\section{L'acquisition des pronoms en L1. Le cas d'enfants à développement typique et d'enfants à développement atypique}

Caroline Rossi et Laurence Vincent-Durroux

\section{OpenEdition}

Journals

Édition électronique

URL : https://journals.openedition.org/corela/14463

DOI : 10.4000/corela. 14463

ISSN : 1638-573X

Éditeur

Cercle linguistique du Centre et de l'Ouest - CerLICO

\section{Référence électronique}

Caroline Rossi et Laurence Vincent-Durroux, « L'acquisition des pronoms en L1. Le cas d'enfants à développement typique et d'enfants à développement atypique ", Corela [En ligne], HS-35 | 2022, mis en ligne le 17 janvier 2022, consulté le 24 janvier 2022. URL : http://journals.openedition.org/corela/ 14463 ; DOI : https://doi.org/10.4000/corela.14463

Ce document a été généré automatiquement le 24 janvier 2022.

\section{cc) (1) (ㅇ)}

Corela - cognition, représentation, langage est mis à disposition selon les termes de la licence Creative Commons Attribution - Pas d'Utilisation Commerciale - Partage dans les Mêmes Conditions 4.0 International. 


\title{
L'acquisition des pronoms en L1. Le cas d'enfants à développement typique et d'enfants à développement atypique
}

\author{
Caroline Rossi et Laurence Vincent-Durroux
}

\section{Introduction}

1 Cet article porte sur l'acquisition des pronoms, d'une part chez des enfants «tout venant », d'autre part chez des enfants à développement atypique du fait d'une surdité profonde ou de troubles spécifiques du langage (TSL). La famille de marqueurs auquel cet article est consacré, les pronoms, est d'une grande complexité en discours car elle combine la morphologie, la syntaxe, la pragmatique, le discours, ainsi que l'influence de l'input et de la qualité de l'interaction (Salazar Orvig \& Morgenstern, 2015 : 155). En outre, au plan cognitif, elle nécessite une capacité à gérer le lien anaphorique, qui présente une particularité a priori difficile pour les enfants : l'abstraction. En effet, le pronom « reprend en occultant » (Cotte, 2008, § 43) et virtualise le terme référent : « le pronom conserve en lui le syntagme initial mais il occulte son identité et il le virtualise " (Cotte, 1996: 273). Nous nous concentrons sur les premiers usages des pronoms et sur ceux, plus tardifs, que l'on trouve dans les propositions subordonnées ou dans les structures clivées chez des enfants de 6-7 ans (Aravind et al., 2017). Nous tentons de répondre aux questions suivantes : quelles sont les étapes de l'acquisition des pronoms en L1 et quelles en sont les difficultés pour les enfants à développement atypique? Nous adoptons une approche interactionniste et constructionniste (Morgenstern, 2006 : 16), qui s'intéresse aux étapes de l'acquisition du langage ainsi qu'aux processus et aux facteurs qui permettent d'en rendre compte. Nous combinons des données de littérature et des analyses en contexte de corpus oraux recueillis auprès d'enfants. L'article se fonde sur des travaux portant sur deux langues : le français et l'anglais. Nous commençons par présenter les principales caractéristiques de 
l'acquisition chez des enfants à développement typique, en allant d'un panorama des premiers usages à la présentation des principales étapes développementales décrites dans la littérature : cette première partie intègre les premières formes de pronoms personnels, possessifs et démonstratifs afin de situer les différentes acquisitions et d'essayer de cerner des processus à l'œuvre. Dans une seconde partie, nous effectuons un gros plan sur l'acquisition des pronoms personnels et sur les difficultés spécifiques de cette acquisition chez les enfants à développement atypique. Nous nous concentrons sur ces pronoms pour leur rôle fondateur dans le développement typique mais aussi parce que ce sont les pronoms étudiés dans diverses situations de développement atypique.

\section{Première partie : vue d'ensemble des phénomènes observés dans le développement typique}

2 Pour présenter tout d'abord quelques points de repère développementaux, nous nous appuyons sur des données de référence issues de tests et de données spontanées. Ces données sont particulièrement utilisées pour décrire l'acquisition précoce, qui nous occupe ici ; il faut cependant signaler, en complément, que d'autres techniques comme l'oculométrie, l'électro-encéphalogramme, l'amorçage ou le regard préférentiel permettent d'aborder le développement de la complexité syntaxique, et notamment de mieux comprendre la nature des premières constructions verbales, et d'évaluer leur degré d'abstraction (Lieven, 2014).

\section{Panorama des premiers usages de formes pronominales chez l'enfant anglophone et francophone}

3 Le Child Development Inventory (CDI) et sa version française, l'inventaire français du développement communicatif (IFDC) se déclinent en deux versions, selon l'âge des enfants: mots et gestes de 8 à 18 mois, mots et énoncés de 16 à 30 mois. Ces instruments de mesure ont été utilisés auprès de milliers de familles pour recueillir des données susceptibles de faire émerger des tendances. En tant que tels, ils peuvent nous donner des indices de la prévalence de certains comportements langagiers ou prélangagiers dans une population d'enfants à âge ou niveau de développement égal (Naigles \& Hoff-Ginsberg, 1998). Il faut préciser que ces instruments prolongent l'analyse de productions spontanées : ces dernières constituent le point de départ de l'élaboration des listes qui composent de tels instruments (listes d'items sur la base desquelles les mères décrivent les productions de l'enfant). Les inventaires sont donc le reflet de l'usage, tel qu'il peut être représenté aussi dans des suivis longitudinaux ou dans les tests de langage (Fenson et al., 1993 ; Kern \& Gayraud, 2010 pour la version française). Enfin, l'établissement récent d'adaptations linguistiques normées des inventaires américains en fait un excellent point de départ pour la comparaison interlangues ${ }^{1}$. Des chercheurs de l'université de Stanford ont ainsi développé et mis en ligne un outil permettant d'interroger librement les données issues de plus de 80000 inventaires administrés dans 29 langues différentes et de visualiser les résultats (Frank et al., 2017) ${ }^{2}$ : les données présentées ici sont issues de la consultation des inventaires américains et français. Si les inventaires évaluent le vocabulaire compris (réceptif) et produit (productif), nous nous intéressons exclusivement ici à la production, puisque 
les données utilisées pour nos analyses qualitatives sont des données de production. D’autre part, étant donné que les premières formes pronominales ne sont que peu répertoriées dans les inventaires qui concernent les plus jeunes enfants (la version "mots et gestes", proposée aux parents de nourrissons entre 8 et 18 mois) nous ne considérons ici que les inventaires «mots et phrases» et donc des données d'enfants âgés de 16 mois et plus. Nous nous concentrons sur les formes qui apparaissent dès les premiers énoncés : les pronoms personnels et possessifs d'une part, et les démonstratifs de l'autre.

\subsection{Les pronoms personnels et possessifs : quelques caractéristiques qui montrent l'importance du dialogue}

Des requêtes effectuées sur WordBank (cf. ci-dessus et note 2) pour une sélection des formes pronominales issues de l'inventaire produisent les résultats présentés dans les figures suivantes (Figures 1 et 2), où l'axe vertical indique la proportion de l'échantillon qui produit les formes et l'axe horizontal l'âge des enfants en mois. Les proportions facilitent la comparaison entre des jeux de données de taille différente : plus de 8000 enfants pour l'anglais américain et plus de 800 enfants pour le français.

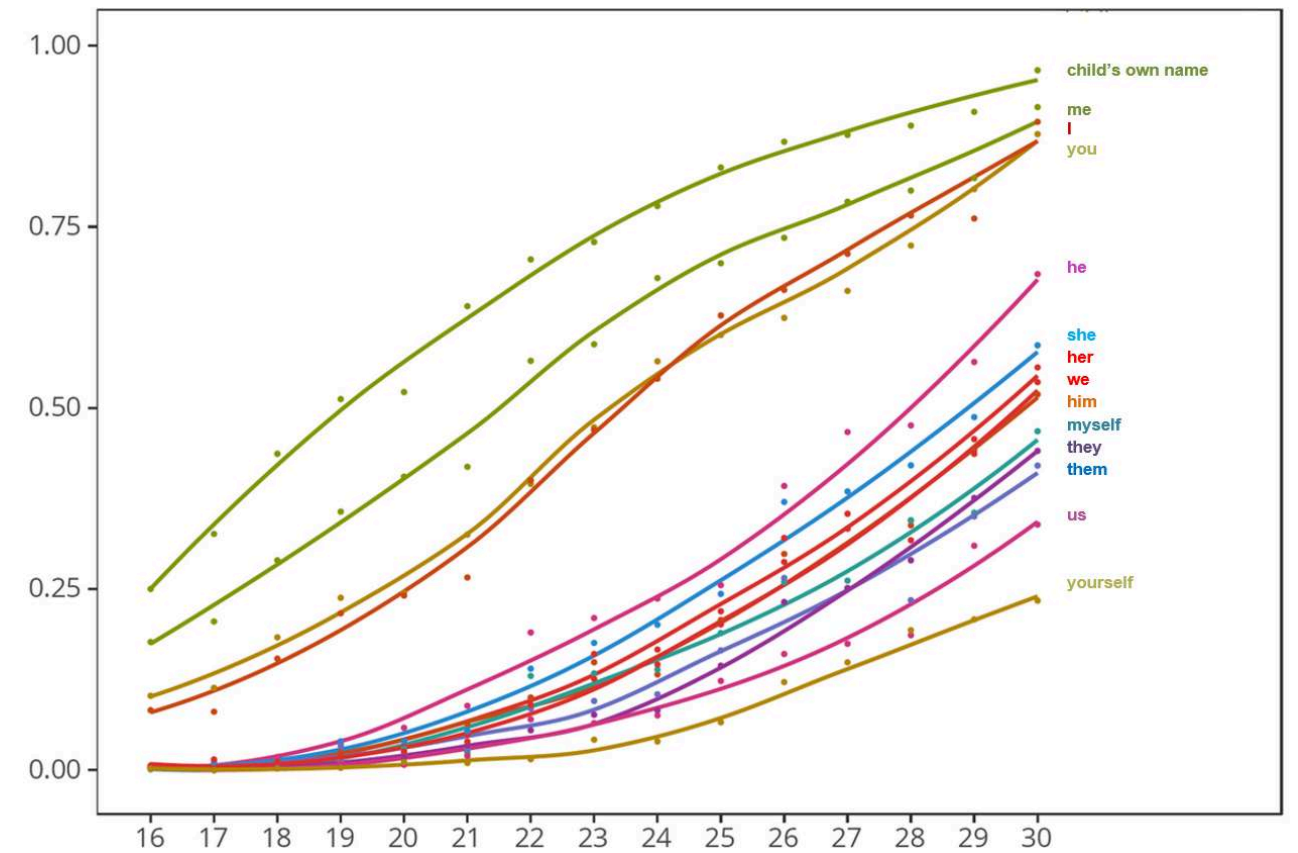

Figure 1 : taux d'emploi des pronoms personnels et du prénom en anglais américain, selon l'âge (en mois).

Plusieurs tendances se dégagent de ces courbes. Nous avons ajouté le prénom de l'enfant pour fournir un étalon sur les usages de la référence personnelle : on constate ainsi tout d'abord que l'enfant utilise son prénom très tôt et que les pronoms constituent une acquisition plus tardive, qui ne remplace pas complètement l'usage du prénom, même à 30 mois. En effet, presque tous les enfants produisent leur prénom à 30 mois (97\%), alors que le pronom de première personne « $I »$ ne concerne que $88 \%$ de la cohorte au même âge. Les pronoms singuliers apparaissent en premier, le pluriel est plus tardif : un peu plus d'un quart des enfants produisent « they» et « them » à 30 mois. La première personne est utilisée très tôt, mais par certains enfants seulement, et il s'agit probablement d'usages dans des expressions figées ou de reprises de l'input 
produites en bloc (souvent appelées holophrases). Les courbes correspondant à la première et à la deuxième personne sont très proches, montrant l'importance du dialogue. On remarque aussi que la première forme plurielle à être utilisée est également un pronom de dialogue : il s'agit de la première personne du pluriel «we », qui concerne près de la moitié des enfants en fin de période.

Les données d'inventaires français font ressortir les mêmes tendances, avec un décalage plus marqué entre "moi » et les autres formes, comparativement à l'anglais (Figure 2). Il faut cependant remarquer que «toi » n'est pas répertorié ici, et que les productions de «tu » et « vous » sont évaluées ensemble alors que la seconde forme est généralement considérée comme plus tardive que la première. Il existe également un écart plus important en français entre la forme tonique «moi », qui est produite par la moitié des enfants francophones autour de 24 mois, et le pronom « je » qui ne concerne la moitié de l'effectif qu'à 30 mois.

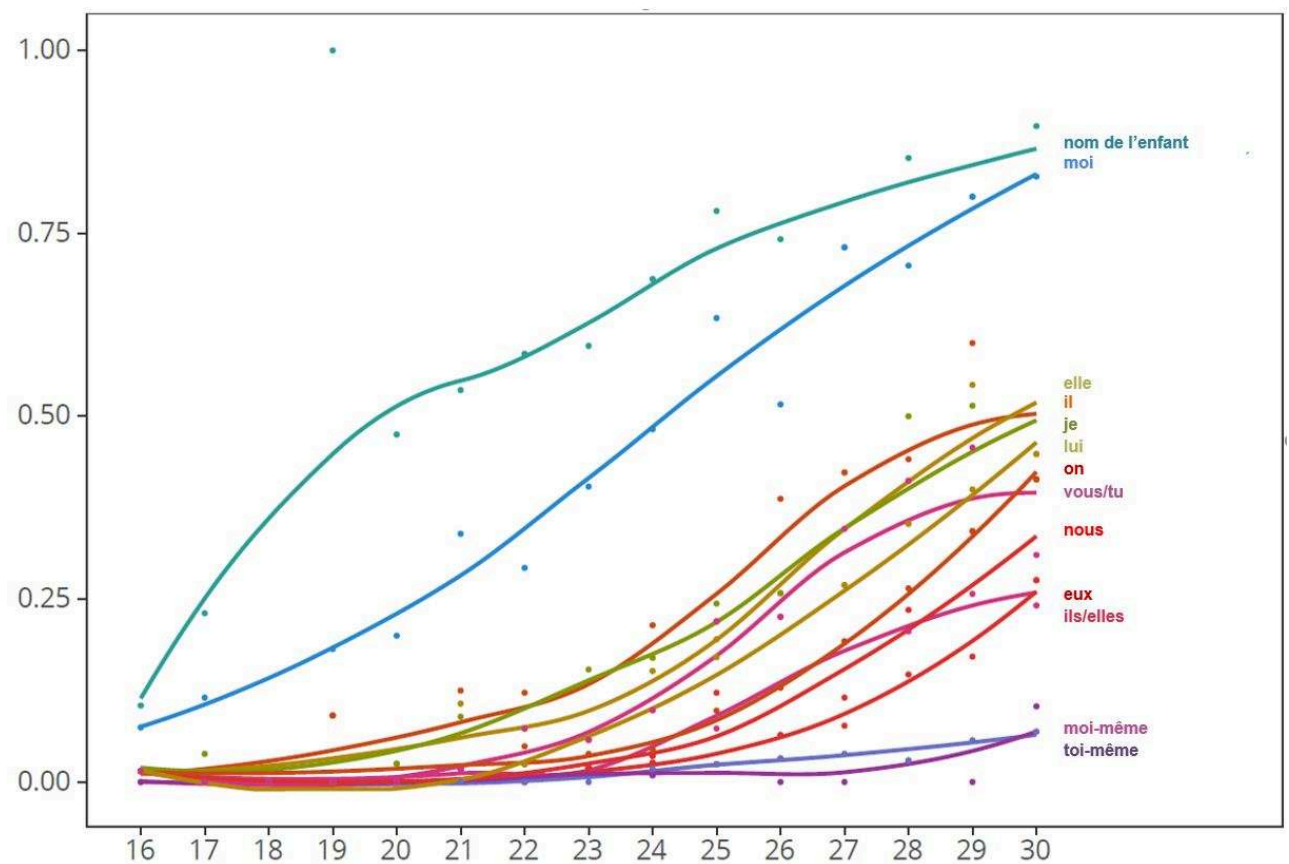

Figure 2 : taux d'emploi des pronoms personnels et du prénom en français selon l'âge (en mois).

Dans l'ensemble, on passe de formes qui ne sont pas observées ou pas considérées par tous les observateurs comme produites de manière spontanée ${ }^{3}$ à des productions attestées dans près de la moitié de l'échantillon autour de 30 mois, sauf pour les pronoms pluriels ou réfléchis. Les formes qui ne sont pas toujours reconnues comme pronoms peuvent correspondre à des syllabes de remplissage ou «fillers », ainsi qu'à la production d'holophrases, et elles expliquent que les résultats présentés dans la littérature soient parfois contradictoires: «il a généralement été observé que les pronoms de $1^{\text {ère }}$ personne apparaissent avant les pronoms de $2^{\text {ème }}$ personne (Bain, 1936 ; Clark, 1978 ; Loveland, 1984 ; Qi 2005). [...] L'émergence de ces pronoms dans des expressions figées explique sans doute que parfois, le pronom de $2^{\text {ème }}$ personne semble émerger avant le pronom de $1^{\text {ère }}$ personne (cf. Sabeau-Jouannet, 1975).» (Caët, 2013 : 26). La répétition de l'input, qui constitue une étape de l'acquisition, pourrait expliquer que l'enfant, à un moment donné, produise plus de pronoms de $2^{\text {ème }}$ personne que de $1^{\text {ère }}$ personne, en rapport avec le fait que, dans son discours, l'adulte réfère plus souvent à l'enfant qu'à lui-même (Salazar Orvig \& Morgenstern, 2015 : 159). 
8 Les pronoms possessifs constituent un autre ensemble de formes personnelles qu'il faut, si l'on adopte le point de vue de l'usage, considérer avec les pronoms personnels. En effet, la possession correspond à un besoin communicationnel précoce de l'enfant (voir par ex. Tomasello, 1998 : 349) et les premiers usages de «moi » sont indissociables de l'expression de la possession avec «à moi » : dans les deux cas, on a la trace d'un positionnement énonciatif fort. La référence à soi en tant que possesseur est très présente chez les enfants pendant la deuxième année, et la variété des marqueurs et des formes fléchies utilisés en français permet de rendre compte d'erreurs repérables chez les enfants francophones (Collombel-Leroy \& Morgenstern, 2012). Autre différence importante : alors qu'en français la forme tonique « moi » s'emploie au même titre que le pronom «mien » («à moi » versus « le mien »), en anglais c'est le pronom " mine » qui sera utilisé presqu'exclusivement pour marquer la possession en première personne (aux côtés des formes adjectivales qui existent aussi en français). Ces premiers constats permettent-ils de rendre compte des tendances observables sur les courbes ci-dessous, qui correspondent aux résultats produits par une recherche sur les pronoms et adjectifs possessifs dans Wordbank, en anglais américain (Figure 3) puis en français (Figure 4)?

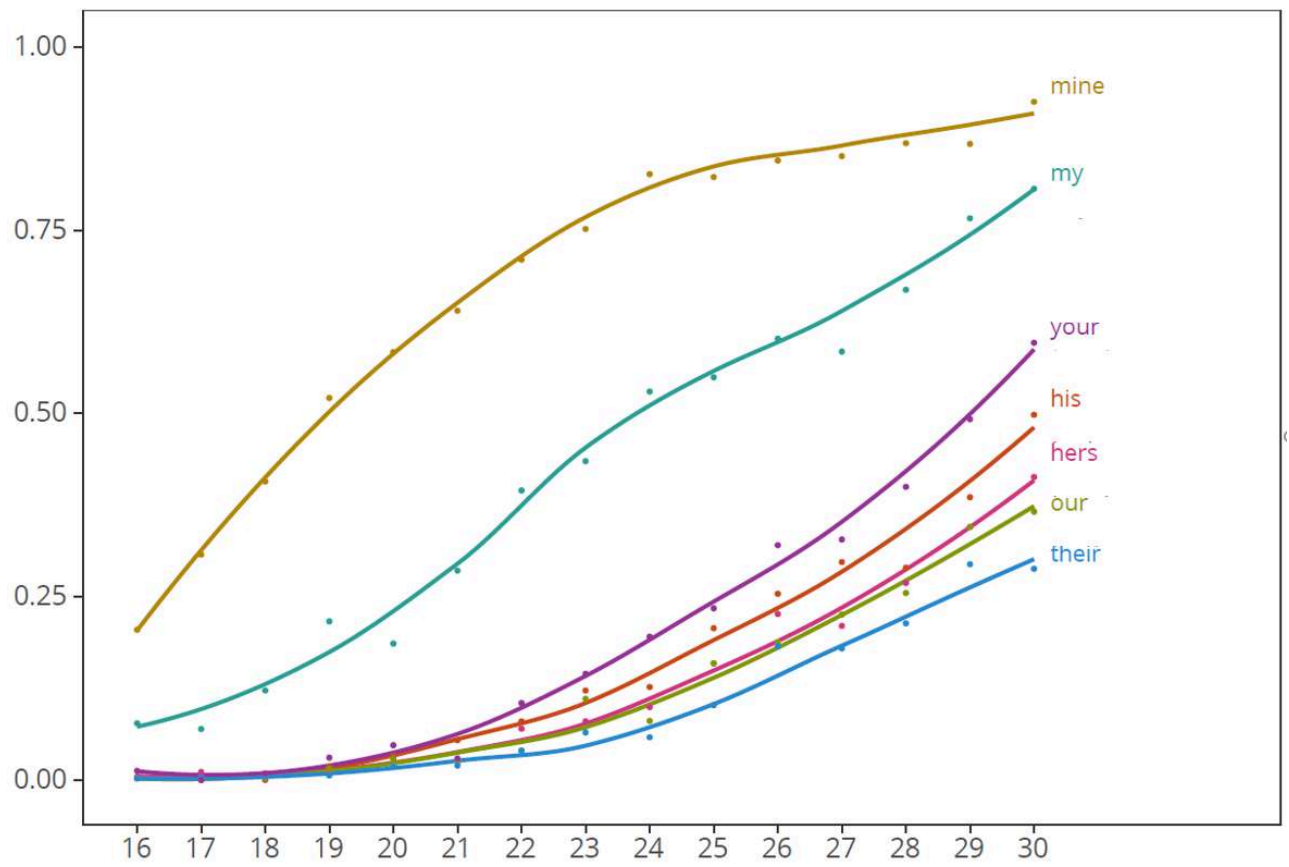

Figure 3 : taux d'emploi des possessifs selon l'âge (en mois) en anglais américain. 


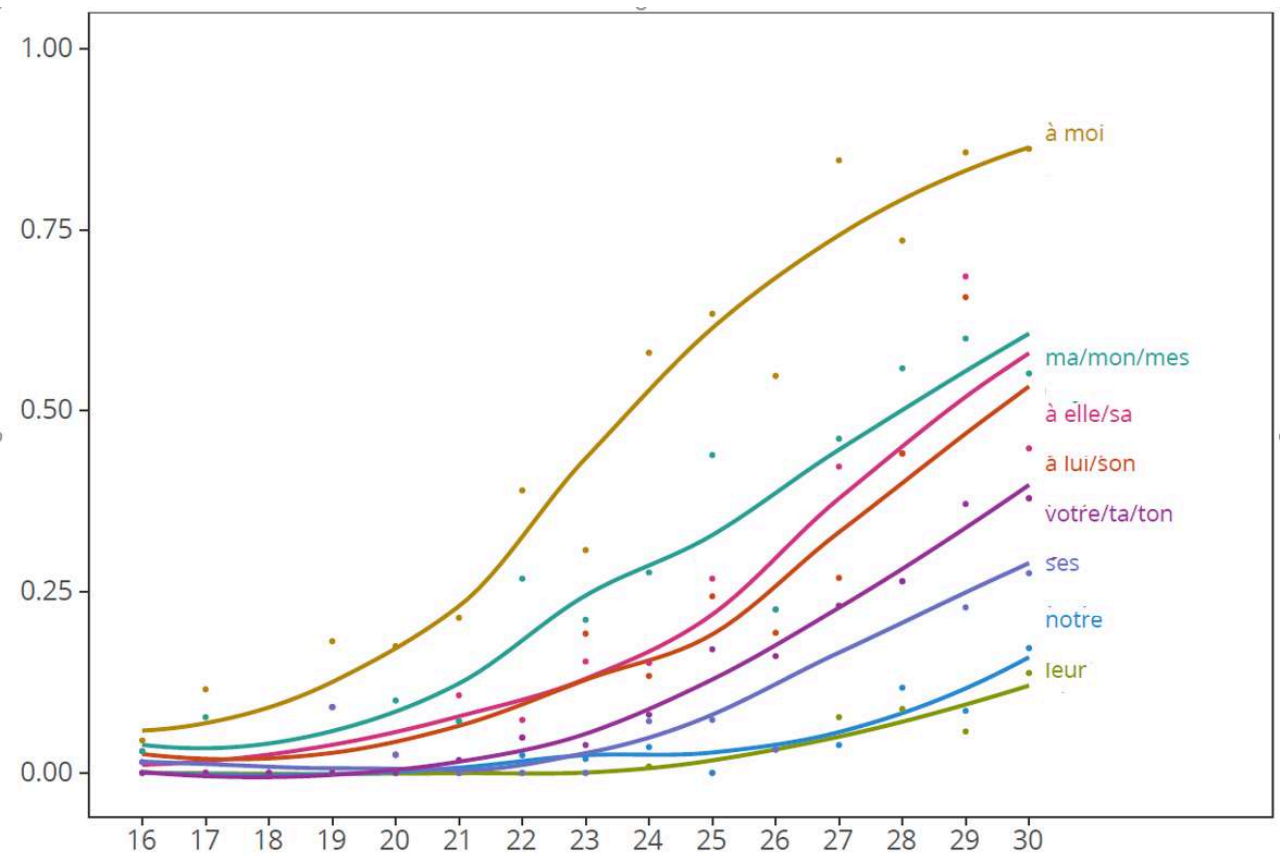

Figure 4 : taux d'emploi des possessifs selon l'âge (en mois) en français.

9 Les courbes montrent notamment que «mine » est appris plus tôt que «à moi »: 75\% des enfants américains le produisent à 22 mois alors qu'au même âge, un peu moins de la moitié des enfants français produisent «à moi ». Cette tendance pourrait être liée à la concurrence entre "moi » et «mien» en français, la seconde forme n'étant pas répertoriée dans les inventaires français. De même, la production généralement plus tardive des formes fléchies françaises («ma », «mon », mes ») confirme qu'il y a là une complexité plus grande qu'en anglais, où une seule forme est utilisée.

\subsection{Les pronoms démonstratifs}

10 La valeur de localisation qui sous-tend la majorité des emplois de démonstratifs est probablement tout aussi primordiale que la possession (Slobin, 1985, in CollombelLeroy \& Morgenstern, 2012: 61). Pourtant, les courbes ci-dessous montrent qu'à 30 mois, les démonstratifs sont globalement moins maitrisés (moins d'enfants les produisent) que les possessifs. 


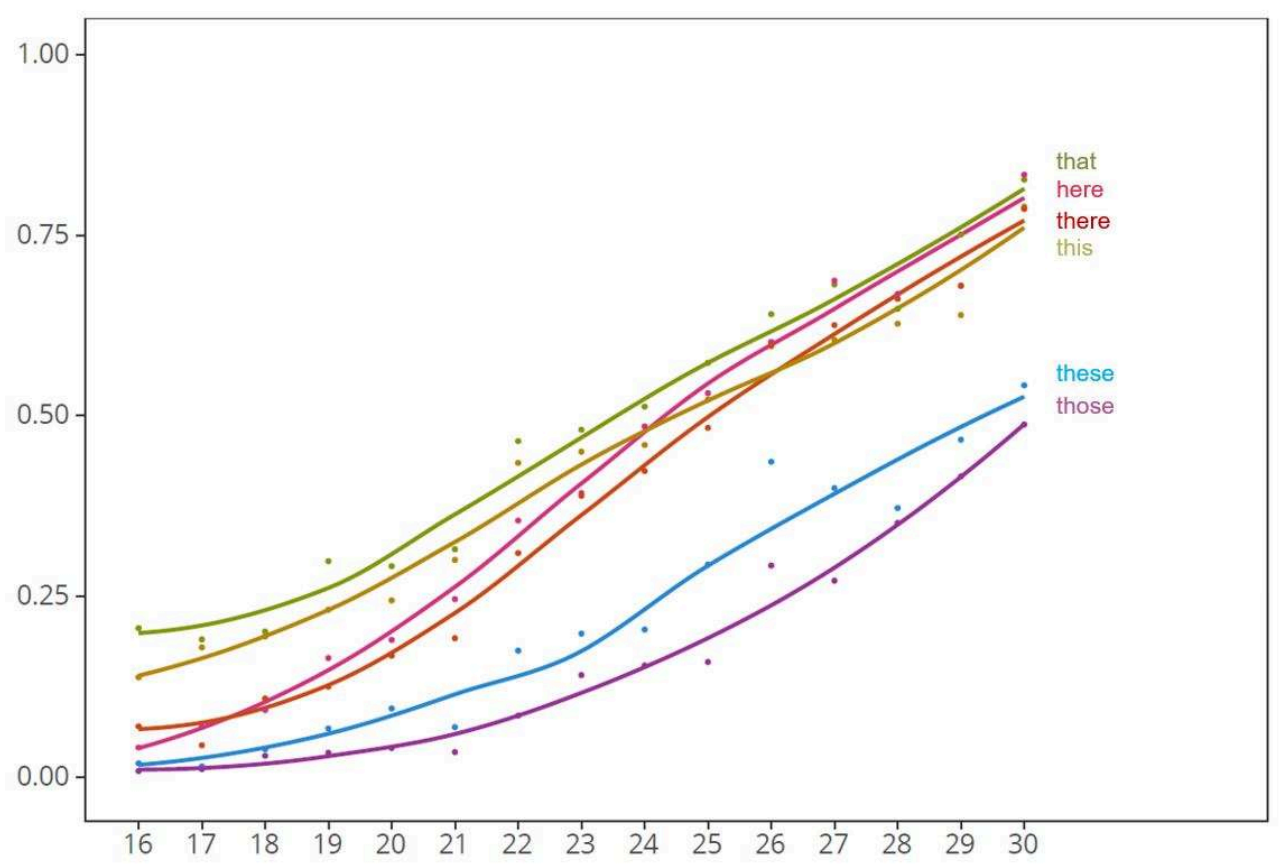

Figure 5 : taux d'emploi des démonstratifs ${ }^{4}$ selon l'âge (en mois) en anglais américain.

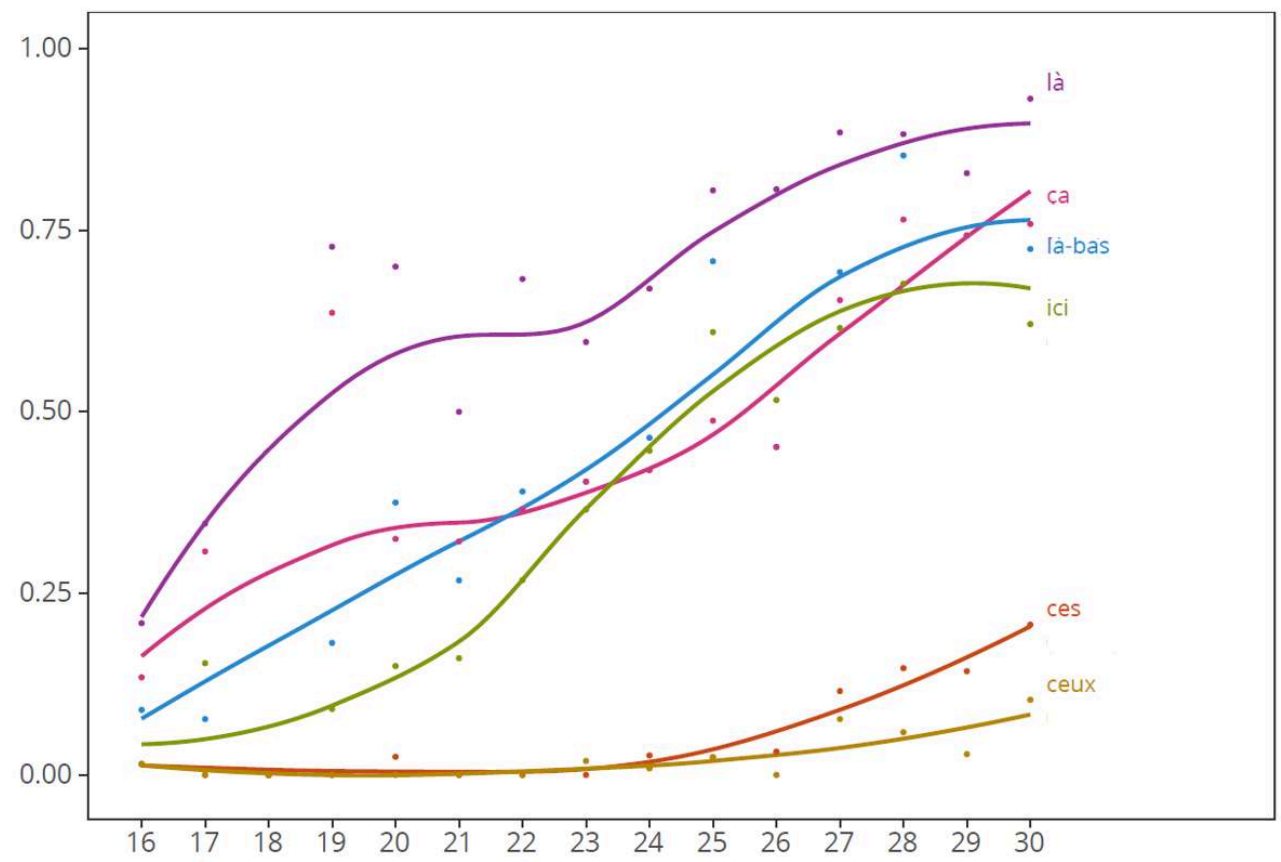

Figure 6 : taux d'emploi des démonstratifs selon l'âge (en mois) en français.

11 Dans l'ensemble, on retrouve la tendance à produire le pluriel plus tard, même si elle est beaucoup plus affirmée en français. A l'inverse, on constate que « ça », « là » et « làbas " correspondent à des productions plus précoces en français: 60\% des enfants produisent «là » à 20 mois, alors que moins de $20 \%$ des anglophones produisent " there » au même âge, ce qui suggère que les marqueurs n'ont pas les mêmes valeurs et sont probablement utilisés dans des contextes en partie distincts. 


\section{Le développement des pronoms chez l'enfant}

12 La revue de littérature que nous présentons ici n'est pas impartiale: elle se fonde principalement sur les travaux menés par des chercheuses et chercheurs travaillant avec les outils des linguistiques fonctionnalistes et basées sur l'usage. Ces chercheurs analysent le plus souvent des données spontanées pour proposer des études de cas. Des situations d'interaction naturaliste parent-enfant ou entre enfants, ou d'autres, plus contraintes car intégrant la production collective de récits à partir d'amorces, sont par exemple comparées avec des situations d'interaction entre adultes (par ex. Martinot, 2005). L'analyse des journaux parentaux est encore utilisée, souvent pour compléter les données enregistrées: c'est notamment le cas de l'étude de Rowland (2007) sur les questions en wh-.

13 Les études qui comparent des suivis d'enfants anglophones et francophones sont relativement rares. Celle de Girouard et al. (1997) est assez ancienne, mais présente l'intérêt d'être faite à grande échelle (12 enfants par langue), à partir de tests standardisés et de quinze minutes de jeu libre. Cette étude nous renseigne principalement sur l'ordre d'acquisition des pronoms personnels en production ( $1^{\text {ère }}$ puis $2^{\text {ème }}$ puis $3^{\text {ème }}$ personnes), et sur les difficultés repérées chez les enfants francophones dans la compréhension des pronoms de $3^{\text {ème }}$ personne si le discours ne leur est pas directement adressé. Les analyses de Thordardottir (2005) sont basées sur des échantillons de production de 37 enfants monolingues de Montréal (19 francophones et 18 anglophones) âgés de 20 à 47 mois, et constituent une référence qui a notamment été utilisée dans l'élaboration des inventaires français du développement communicatif (Kern \& Gayraud, 2010). Ces analyses permettent de dresser un panorama de l'acquisition des flexions grammaticales du français, en utilisant le seuil des $50 \%$ de la cohorte et en considérant le nombre de mots différents utilisés avec la même flexion dans trois groupes d'âge (24, 32 et 43 mois en moyenne). Elles montrent notamment que la marque du genre sur les pronoms (hors pronoms personnels) n'est acquise qu'en fin de période. Enfin, une étude plus récente (Caët, 2013) se fonde sur deux suivis longitudinaux par langue (anglais américain et français) et sur le discours adressé à l'enfant. Les résultats indiquent une influence possible de la fréquence de formes et de constructions similaires dans l'input sur les formes non-standard de référence à soi et à l'interlocuteur produites par les enfants. Ils montrent également une diversité d'emplois peu décrite dans la littérature.

14 Au-delà de ces apports particuliers, des points communs se détachent qui permettent de caractériser les étapes développementales et les processus à l'œuvre dans l'acquisition des pronoms.

\subsection{Les étapes}

15 On trouve chez le tout jeune enfant des productions originales, qui ne se conforment pas à celles de l'adulte (décrites notamment par Morgenstern, 1995; 2003 ; Budwig, 1995) : pour plusieurs auteurs, elles sont la trace de l'appropriation progressive du système (Morgenstern, 2006 ; 2012 ; Caët, 2013). Deux types sont à relever :

16 - les « proto-formes » : Ø en position sujet; des formes transitionnelles (fillers) 
17 - les formes inhabituelles, telles que des noms propres remplaçant les pronoms personnels (nom de l'enfant ou de son interlocuteur), le renversement pronominal (

Ces usages ont été régulièrement observés et des explications ont été proposées. Par exemple, pour les confusions de formes sujet/objet, Budwig (1995) a observé chez des enfants anglophones des différences liées au degré d'agentivité ; Deutsch \& Budwig (1983) ont montré à partir de suivis longitudinaux d'enfants anglophones qu'il existait un contraste entre des énoncés du type «[nom de l'enfant] pencil » et «my pencil », le premier ayant une fonction indicative (désignation d'un objet) alors que le possessif exprime la volition (volonté de récupérer un objet qui est utilisé par quelqu'un d'autre, par exemple).

19 Les possessifs constituent en réalité un cas intéressant à plus d'un titre, notamment parce qu'ils permettent de suivre l'acquisition progressive des marqueurs pronominaux. La confusion de l'adjectif et du pronom possessifs est fréquente en anglais, avec un usage adjectival premier: cela apparaît bien dans les inventaires, puisque seul l'adjectif est répertorié dans la version 8-16 mois, « mots et gestes ». On en trouve aussi des exemples chez Tomasello (1998), avec le cas d'un enfant qui utilise les deux formes en position adjectivale : « mine pillow» (Travis 1;06,27), «my book» (Travis $1 ; 07,08) ;$ « drink mine tea » (Travis 1;08,19).

20 Lorsque les formes pronominales deviennent productives, c'est la position préverbale qui est utilisée le plus souvent: les premiers pronoms sont donc sujets des premiers énoncés (pronoms personnels à hauteur de $66 \%$ et impersonnels à $22 \%$ dans les données de Martinot, 2005: 44). La forme objet "me» apparaît également en position préverbale: plusieurs auteurs font l'hypothèse d'une reprise en bloc du discours adressé, notamment pour la formule « let me do it » (Kirjavainen et al., 2009, in Caët \& Morgenstern, 2015 : 176). Enfin, lorsque les pronoms commencent à être utilisés dans la complémentation verbale, Childers et Tomasello (2001) envisagent qu'ils jouent un rôle facilitateur dans l'acquisition des constructions transitives.

\subsection{La deixis et l'ancrage spatial des premières productions}

21 Pour situer et localiser, l'attention conjointe est souvent matérialisée plus clairement par les adultes dans les interactions avec le jeune enfant : le rituel du coucou-caché en est un exemple (Rossi, 2017). Les formes qui sont alors produites dans le discours adressé sont reprises assez tôt par l'enfant: les données d'inventaires présentées cidessus le montrent tant pour les démonstratifs («celui-ci / celui-là ») que pour les adverbes correspondants (« ici / là » ou « là-bas »). Elles montrent aussi une tendance à utiliser des marqueurs déictiques, qui semble plus frappante chez les enfants francophones, probablement parce qu'ils utilisent d'abord et surtout le couple «ça/là » avec des fonctions plus étendues que celles du couple «this/that » en anglais.

D'autre part, les pronoms permettent de reprendre un référent nominal souvent présent dans le contexte interactionnel, et dont la désignation est une façon de le localiser, comme le montrent les exemples ci-dessous (tirés de Clark, 1998). On voit que pour localiser un référent nominal, on en parle de façon répétée et que c'est dans ce contexte que les formes pronominales émergent peu à peu.

(1) cassées les jambes (= elles sont cassées les jambes) P (20 mois)

(2) fermée la fenêtre (= elle est fermée la fenêtre) P (21 mois)

(3) a passé tram (= il est passé, le tram) L (33 mois) 
Les premières formes pronominales sont d'abord omises, au profit de référents nominaux situés en fin d'énoncé : on passera ainsi de «fermée la fenêtre » à « Elle est fermée, la fenêtre ». En français comme en anglais, la nécessité de remplir la position sujet n'est initialement pas prise en compte.

En anglais, l'analyse de productions spontanées a aussi montré que pour poser le référent stable, objet de l'attention conjointe, l'enfant construisait d'abord un repère en début de phrase (les exemples ci-dessous sont ceux de Diessel, 2004) :

(4) that's Daddy

(5) that's Weezer

(6) that's my chair

(7) that's him

(8) that's a paper too

(9) that's Mark's book

(10) that's too little for me

Plus tard, les compléments locatifs restent fréquents mais ils se situent plutôt à droite du verbe et ils marquent les débuts de la complémentation verbale. Dans son analyse de récits d'enfants francophones de 6 ans, Martinot (2005: 43) remarque ainsi qu'en moyenne une phrase sur 5 comporte un complément locatif.

Il s'agit probablement là d'une première étape, et l'on peut remarquer que la deixis en comporte au moins deux, qui ne sont pas toujours distinguées : elle consiste, d'une part, à poser un référent stable objet d'attention conjointe (qui peut être mis en relief, mais aussi faire l'objet d'une reprise pronominale) et d'autre part, elle implique que le locuteur soit capable de prendre la perspective de l'autre, en lien avec les capacités en théorie de l'esprit (voir par ex. Gundel \& Johnson, 2013, in Salazar Orvig \& Morgenstern, $2015: 168)$. On peut faire aussi une lecture spatiale de ce deuxième point : situer le point de vue de l'autre, et l'adopter, c'est se mettre à la place de l'autre. Le développement cognitif est progressif : selon Clark (1978), la deuxième personne est plus difficile à appréhender car elle fait davantage varier les référents, mais il faut mentionner aussi la perspective égocentrique du jeune enfant (Sinclair-de-Zwart, 1969) et préciser que dans les interactions précoces l'attention se porte le plus souvent sur les événements auxquels les enfants participent (Budwig, 1990).

Ces premiers constats sur les étapes développementales et le caractère fondateur des opérations énonciatives liées à la deixis appellent des précisions sur au moins deux éléments : tout d'abord, le passage progressif de productions où les pronoms ne sont pas systématiquement produits à d'éventuelles répétitions, avant d'atteindre la concision, et d'autre part, la construction de la référence personnelle dans des interactions où ce que Benveniste appelait les «pronoms de dialogue » se répondent et se mêlent.

\subsection{Répéter puis impliciter : de la redondance à la concision}

Les énoncés redondants sont bien plus fréquents chez les enfants, en vertu d'un principe qui peut s'énoncer ainsi: "le sens est déplié, explicité » alors qu'il est condensé dans le discours adulte (Martinot, 2005 : 145; ce constat porte, comme cidessus, sur l'analyse de narrations enfantines produites par des enfants de 6 ans). 
Dans l'ensemble, la complémentation verbale est une acquisition très progressive, avec des patrons de plus en plus abstraits, peu à peu associés à de nouveaux verbes (Childers \& Tomasello, 2001 ; Lieven, 2014)

Sekali (2012) décrit un effet accordéon (« concertina effect ») : à partir des travaux de Diessel (2004) sur l'intégration progressive de constituants phrastiques, elle fait apparaître cet effet accordéon dans l'acquisition des causales en "parce que ». Cette expansion progressive caractérise d'abord une seule et même situation :

(11) maman elle donne le lait à Côme parce que là il pleure (Madeleine 32 mois)

(12) tu peux prendre ma cuillère parce que moi...5 ( (Madeleine 32 mois)

31 Les exemples ci-dessus sont ceux de Sekali (2012) et concernent un suivi longitudinal issu du corpus CoLaJe ${ }^{6}$ (Morgenstern \& Parisse, 2007). Ils montrent une bonne maitrise des pronoms personnels singuliers chez un enfant de 32 mois, ainsi qu'un usage, en début de proposition complétive, de marqueurs déictiques ( moi » et « là ») qui semblent redondants mais constituent aussi des points de repère du même type que ceux décrits ci-dessus dans des phrases simples.

Dans un second temps, il y a intégration de deux situations :

(13) elle s'est noyée parce qu'elle avait pas de brassards (Madeleine 49 mois).

L'intégration se fait ici sans redondance, et à l'aide de deux pronoms de troisième personne qui sont en outre coréférentiels.

\subsection{Construire la référence à soi}

\subsubsection{Le renversement pronominal}

Nous l'avons vu dans les données d'inventaires, la référence à soi n'est pas une évidence, surtout dans le discours adressé à l'enfant, où les mères ont tendance à s'effacer et à faire la part belle au discours sur l'enfant, ainsi que le montre la figure 7 ci-dessous.

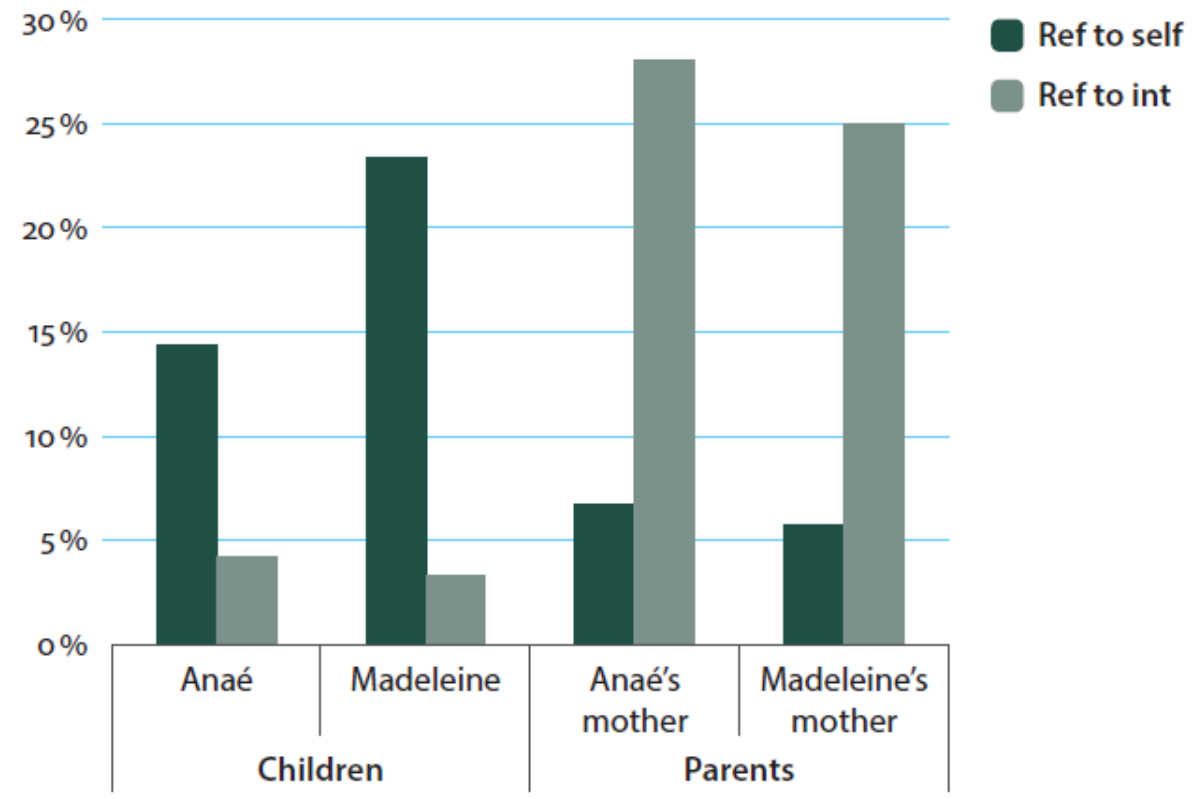

Figure 7. Proportion moyenne des énoncés qui font référence à soi ou à l'interlocuteur, chez deux mères et deux enfants étudiés de 18 à 39 mois (source : Caët \& Morgenstern, 2015 : 178) 
dans ce contexte que de nombreux enfants produisent ce que l'on a coutume d'appeler des renversements pronominaux, lorsqu'ils utilisent la marque de la deuxième personne pour se désigner. Plusieurs facteurs ont été avancés pour rendre compte des productions illustrées dans le dialogue ci-dessous entre Anaé ${ }^{7}$ âgée de 26 mois et sa mère (Caët \& Morgenstern, 2015 : 190) :

(14) La mère : attention attention tu rentres bien ta tête

rentre bien ta tête

La mère : tu montes tes fesses

La mère : vas-y rentre ta tête [...] Bravo !

Anaé : tu remontes tes fesses (en rigolant)

Ces facteurs ne sont pas mutuellement exclusifs : à la reprise en écho du discours adressé, qui s'illustre bien ici, s'ajoute ainsi la difficulté que peut avoir le jeune enfant pour saisir le contenu sémantique des pronoms de première et de deuxième personne, comme le changement de perspective qu'ils permettent (voir Caët \& Morgenstern, 2015 : 175 et sq. pour une analyse détaillée).

\subsubsection{Les clitiques dans l'acquisition du français}

Outre les renversements pronominaux que l'on n'observe pas chez tous les enfants, nous avons remarqué que l'acquisition du pronom de première personne était délicate et plus tardive que celle du prénom, qui est d'abord utilisé par l'enfant pour se désigner. Le premier élément d'explication est le manque de saillance de cette forme dans l'input: moins fréquent que la référence à l'enfant (cf. supra), le «je» est également dépourvu d'accent. Ainsi, et d'une manière plus générale, les clitiques ne peuvent pas être produits de façon isolée car ils ne sont pas porteurs d'accent, et les holophrases intègrent uniquement des pronoms toniques. C'est ce qui explique de l'on trouve d'abord, dans les inventaires parentaux, le prénom puis les pronoms forts en référence à soi, le clitique « je » étant plus tardif.

Ensuite, les clitiques nominatifs sont acquis avant les clitiques accusatifs, et les clitiques réfléchis avant les clitiques non réfléchis (Jakubowicz \& Rigaut, $2000: 127$ et sq., Salazar Orvig et al., 2010). Ainsi, les clitiques accusatifs sont souvent omis dans les premiers énoncés, ce qui peut s'expliquer par leur forme faible et par la complexité syntaxique que leur emploi entraîne. En voici un exemple extrait d'un enregistrement de Madeleine (corpus CoLaJe) à 28 mois, où l'on voit que l'enfant préfère un complément nominal («maman ») au clitique objet « te »:

(15) Madeleine : /ze/ téléphone maman

La mère : ah tu me téléphones?

La redondance, courante à l'oral mais dont nous avons vu qu'elle était plus utilisée par le jeune enfant, concerne davantage les clitiques sujets (comme dans l'exemple 11 cidessus) mais parfois aussi les clitiques objets comme dans cet énoncé d'une petite fille âgée de 30 mois, issu des données de Jakubowicz \& Rigaut (2000: 134) : « on les voit pas, les chaussures ".

Enfin, certains auteurs ont remarqué que les clitiques objets apparaissaient en même temps que l'article défini: si les processus peuvent être rapprochés, c'est qu'ils impliquent tous deux le marquage linguistique de la présupposition (Müller \& Kupisch, 2007). 
41 La figure 8 ci-dessous récapitule les principales étapes développementales discutées jusqu'ici en présentant un inventaire des formes (colonne de gauche) ainsi que des types d'explications qui ont été proposées dans la littérature (colonne de droite).

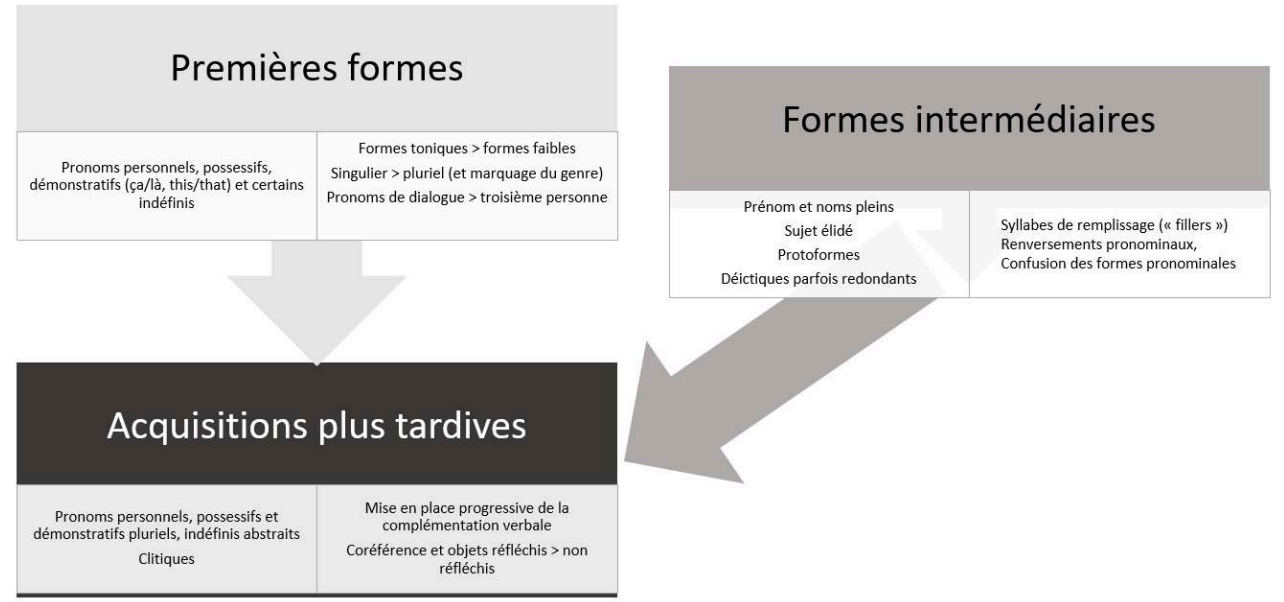

Figure 8. Vue d'ensemble des premières étapes de l'acquisition des pronoms en français et en anglais.

Cette figure montre notamment que les formes toniques sont acquises plus tôt, ainsi que les pronoms de $1^{\text {ère }}$ et 2 ème personnes. Plus tardivement, s'installent la complémentation verbale, la co-référence et les clitiques. Notons que pour ces derniers, les clitiques sujets sont acquis avant les clitiques objets (Salazar Orvig \& Morgenstern, 2015 : 156), ce qui pourrait s'expliquer par la modification de l'ordre canonique sujetverbe-objet dans le cas des clitiques objets (voir paragraphe 3 ci-dessous).

En regard de ces connaissances sur l'acquisition des pronoms chez l'enfant à développement typique, quelles sont les spécificités ou les difficultés rencontrées dans un développement atypique?

\section{Deuxième partie : l'acquisition atypique des pronoms personnels en L1}

Les enfants dont le développement langagier est atypique, soit parce qu'ils manifestent des troubles spécifiques du langage (TSL) ou "dysphasie", soit parce qu'ils sont atteints de surdité sévère à profonde, présentent-ils des particularités dans l'acquisition des pronoms? Nous nous intéressons plus particulièrement ici à l'acquisition des pronoms personnels, d'une part, parce qu'ils sont au fondement de l'acquisition des pronoms (Figure 8), d'autre part, parce que les études portant sur les enfants au développement atypique se focalisent sur cette catégorie de pronoms.

Les sujets avec troubles spécifiques du langage (TSL) ont de graves troubles du développement langagier sans avoir de déficience mentale, neurologique ou sensorielle. Ce contexte est toutefois rapproché de la déficience sensorielle causée par la surdité, car les productions des enfants concernés présentent des similitudes qui pourraient évoquer une cause commune, la surcharge cognitive : ainsi, les difficultés morphosyntaxiques ne seraient pas spécifiques à une pathologie mais plutôt liées à des limitations de capacité de traitement partagées par les enfants concernés (Bourdin et al., 2016). 

la langue orale est majoritairement choisie pour l'enfant sourd de parents entendants (Tomblin et al., 2005). L'acquisition de la langue des signes est non pertinente pour nous car son développement est typique si la langue des signes est présente dans la famille (3 à $4 \%$ des cas de surdité profonde prélinguistique). Nous nous intéressons plus particulièrement au contexte où les enfants sont porteurs d'implants cochléaires (IC), un dispositif chirurgical qui donne accès aux fréquences de la voix et au niveau d'intensité de la conversation. Les enfants représentent $45 \%$ des 12000 implantations réalisées chaque année en France. Grâce à la miniaturisation des éléments numériques $\mathrm{du}$ système implanté, l'implantation est possible avant 12 mois; pour un bon développement du langage, la précocité de l'implantation est recommandée par la Société française d'ORL et de chirurgie de la face et du cou (SFORL, $2018: 7$ ). une revue de la littérature (pour les enfants avec TSL et les enfants avec une surdité moyenne à sévère), d'autre part sur un corpus de données orales spontanées recueillies en situation d'interaction d'enfants sourds profonds de 4 ans porteurs d'implants depuis 36 mois, avec leur parent entendant, en français ou en anglais, langues maternelles. L'étude récente de Bourdin et al. (2016) est particulièrement utile pour notre propos car elle compare les capacités d'enfants avec TSL avec celles d'enfants ayant différents degrés de surdité, en compréhension comme en production.

La mise en évidence des difficultés morphosyntaxiques est le plus souvent obtenue par des tests spécifiques: en compréhension, ce sont des tests d'appariement de phrases orales et d'images (ce qui pose toutefois le problème de la perception auditive); en production, ce sont des tests de répétition (ce qui pose également un problème, celui de faire intervenir la mémoire de travail qui semble déficitaire chez ces enfants, ainsi que nous verrons ci-dessous) et des tests d'amorce à compléter. Plus rares sont les études qui se fondent sur l'analyse de discours spontané.

\section{Résultats obtenus par des tests}

\subsection{Les sujets avec TSL}

Les déficits sont très marqués dans l'acquisition de la morphosyntaxe et en particulier de la morphologie verbale; ils sont relevés dans la plupart des langues: anglais (Leonard, 1998), français (Parisse \& Maillart, 2004), allemand, suédois, néerlandais.

En français, de sérieuses difficultés apparaissent avec les pronoms clitiques (Jakubowicz \& Rigaut, 2000 ; Paradis et al., 2002), dont la faible production constitue même un critère dans le diagnostic des pathologies des TSL (Bourdin et al., 2016 : 162). De même, les structures syntaxiques qui impliquent un mouvement syntaxique complexe, tels les clitiques objet en français, mais aussi le passif, posent des difficultés en compréhension et surtout en production (Cronel-Ohayon, 2004) où on constate un évitement de ces structures. Bourdin $(2016: 147)$ signale qu'à l'âge de 10 ans 6 mois en moyenne, 92\% des cas des enfants avec TSL et $96 \%$ des enfants sourds produisent des syntagmes nominaux plutôt que des pronoms. Cela n'est pas sans rappeler, mais à un âge bien plus tardif ici (10 ans 6 mois, au lieu de 6 ans), la phase de redondance chez les enfants à développement typique ( $\$ 2.3$. ci-dessus), qui « déplient » le sens avant d'aborder une phase d'implicitation. 


\subsection{Les sujets avec surdité moyenne}

51 Les études montrent qu'en français, les enfants ayant une surdité moyenne ont une bonne maitrise de la production des déterminants définis («le», «la», «les») et du clitique nominatif (« il », « elle»), mais pas du clitique accusatif («le», « la», « les») ni du clitique réfléchi («se »), en cohérence avec le fait que ces formes font également l'objet d'une acquisition plus tardive chez les enfants à développement typique (Figure 8). Chez les enfants à développement atypique, des stratégies d'évitement de ces structures complexes sont observées (Tuller \& Jakubowicz, 2004). De fortes variations inter-individuelles sont également relevées (Duchesne et al., 2009), en corrélation avec les caractéristiques du dialogue maternel (longueur des énoncés, qualité des reformulations). Outre le rôle de l'input, il faut noter que les formes les plus difficiles à acquérir (clitiques accusatifs et clitiques réfléchis) sont caractérisées par une antéposition au verbe et donc par une modification du schéma canonique du français. Cette modification pourrait constituer une difficulté pour les enfants sourds (voir paragraphe 3 ci-dessous).

\subsection{Les sujets avec surdité sévère à profonde}

Malgré des résultats souvent dans la norme aux tests globaux, les enfants sourds sévères (perte auditive de 70 à $90 \mathrm{~dB}$ ) à profonds (perte auditive supérieure à $90 \mathrm{~dB}$ ), porteurs d'implants cochléaires, ont des performances inférieures en morphologie et en syntaxe par rapport aux autres composantes langagières (Hallé \& Duchesne, 2015), avec des omissions et des substitutions, même si dans le cas des pronoms clitiques objet, l'omission reste fréquente également chez l'enfant entendant jusqu'à 4 ans (Bourdin, 2015 : 472).

Les difficultés morphosyntaxiques relevées par Jacq et al. (1999), chez des enfants francophones, concernent, par ordre croissant de difficulté: les déterminants, les pronoms clitiques sujets, les pronoms clitiques réfléchis et les pronoms clitiques accusatifs.

54 Il est intéressant de constater qu'en italien, dont la structure syntaxique est similaire au français pour l'emploi des pronoms clitiques, avec antéposition par rapport au verbe, les pronoms clitiques objets de $3^{\text {ème }}$ personne sont omis de façon significative avec deux ans de retard par rapport au groupe témoin entendant, malgré une implantation précoce (Guasti et al., 2012) ; il y a notamment omission des pronoms clitiques dans des tâches de répétition (Caselli et al., 2012; Chilosi et al., 2013) et d'amorces à compléter (Guasti et al., 2012). Enfin des erreurs portant sur le genre du pronom clitique (Chilosi et al., 2013) sont relevées. Ces erreurs de genre pourraient être attribuées aux fines variations vocaliques qui les caractérisent en italien, et donc à des difficultés de discrimination en contexte de surdité, y compris sévère.

\section{Résultats issus de productions spontanées}

L'analyse de données spontanées que nous avons menée après avoir filmé des enfants sourds profonds en France et au Royaume-Uni ${ }^{8}$ permet de faire apparaître un point fondamental que les résultats obtenus par des tests ne mentionnent jamais: la difficulté des enfants sourds dans l'emploi suivi de pronoms. Nous présentons ici une 
partie des résultats obtenus dans le cadre d'une étude préliminaire au projet DEL (Deafness, Early implantation, Language) (Vincent-Durroux et al., 2018).

Évoquons tout d'abord les structures dans lesquelles s'insèrent ces pronoms. Les enfants francophones montrent leur capacité à utiliser correctement la structure cible dans $92 \%$ des cas. En voici quelques exemples :

(16) un ours qui met un pantalon (X METTRE Y)

(17) tortue met chapeau (X METTRE Y)

(18) j'aime bien les cabanes (X AIMER Y)

(19) il mange un poisson (X MANGER Y)

La structure transitive en tant que telle peut être considérée comme réellement maitrisée si le sujet la choisit à dessein lorsque les structures intransitives et transitives co-existent pour un même verbe, comme dans le cas du verbe PARLER («il parle » / « il parle six langues »). Nous en avons des exemples dans nos données avec le verbe AIMER :

(20) j'aime pas (X AIMER)

(21) moi j'aime bien faire des legos (X AIMER Y)

Dans seulement $8 \%$ des cas, à 36 mois post-implant, les deux enfants sourds produisent des énoncés en S V $\varnothing$ alors que la cible est SVO (sujet verbe objet) :

(22) moi je veux pas mettre là-dedans (X X METTRE + $\varnothing+$ Lieu)

(23) on va mettre sur elle (X METTRE $\varnothing+$ Lieu)

(24) comment on met (X METTRE $\varnothing+$ Manière)

(25) on accroche là voilà (X ACCROCHER Ø + Lieu)

(26) Xx mets pas (X METTRE Ø) (xx : syllabes non interprétables)

Dans la plupart des occurrences de ce type, on relève la mention d'un lieu (« là », « làdedans ", " sur elle »), qui, en plus d'être conforme à ce qui est décrit chez les jeunes enfants (§ 2.2. ci-dessus et Martinot, $2005: 43$ ), peut jouer à deux niveaux : d'une part, ce terme occupe une place à droite du verbe, et même si ce n'est pas syntaxiquement celle de l'argument $\mathrm{Y}$, l'énoncé prend ainsi un volume phonologique typique d'un énoncé transitif (en acquisition, il y a souvent association de la prosodie et de la syntaxe, cf. Martel \& Dodane, 2012) ; d'autre part, le lieu situe le référent de Y, ce qui pourrait expliquer que ce dernier, étant localisé, puisse plus facilement faire l'objet d'implicite.

\subsection{L'emploi de pronoms en co-référence ponctuelle}

Les clitiques accusatifs peuvent bien souvent être compris par la situation et / ou par le co-texte. La référence est à trouver dans la situation dans les exemples suivants extraits de nos données :

(27) c'est moi qui l'a encore (à propos d'une carte de Loto)

(28) est-ce que ça lui va (à propos de Monsieur Patate ${ }^{\mathrm{TM}}$, désigné par « lui »)

(29) ah ouai comment on le monte (idem)

61 Le terme co-référent peut aussi apparaître dans le co-texte, notamment en cas de dislocation, du sujet (30) comme de l'objet (31). Dans ces exemples, " elle » et « cette patate » d'une part, « les pieds » et « les » d'autre part, sont co-référents :

(30) elle est rigolote hein cette patate

(31) les pieds on va pas les mettre 
La double complémentation, comme celle du verbe METTRE (METTRE QUELQUE CHOSE

A QUELQU'UN), où le clitique peut concerner les deux compléments («le mettre » / « lui mettre ») n'apparaît pas dans le corpus.

L'un des enfants produit peu de clitiques; en revanche on trouve le pronom " qui ", effectuant une reprise de l'antécédent sujet dans la structure " un S qui V O ", massivement produite dans cet enregistrement :

(32) quand on prend sa douche on le met là

(33) un ours qui joue au ballon

(34) une voiture qui qui joue avec une tortue une tortue qui joue avec sa tortue une tortue qui joue avec la voiture

\subsection{La co-référence suivie}

Le recours à la co-référence dans le cadre d'une chaîne de co-référence (Chastain, 1975) pose-t-il des difficultés particulières aux enfants sourds?

Une co-référence suivie requiert le maintien du lien référentiel « sur du long terme référentiel » (Schnedecker \& Landragin, 2014). Or, la continuité référentielle peut s'avérer difficile chez les sourds profonds, même adolescents, comme cela a été montré pour les déterminants dans les énumérations (Vincent-Durroux, $2014: 41$ ) ou pour l'ancrage temporel de verbes successifs dans une narration (Vincent-Durroux, 2014: 59 sq.).

66 L'analyse du corpus révèle que, chez l'un des enfants francophones (CI-M), la coréférence suivie donne lieu à l'abandon du clitique (énoncés 35 et 36), ce qui n'est pas le cas de l'autre enfant francophone (CI-R) (énoncé 37) :

(35) et ça on va pas mettre ça là-dedans on va $\varnothing$ mettre sur sur elle madame patate mais ça dépasse

(36) on peut la mettre hein on peut la rajouter mais comment on $\varnothing$ met

(37) pour prendre pour prendre sa douche il met ça devant il met ça derrière pour prendre sa douche on le met là

$67 \mathrm{Au}$ fil des énoncés, CI-M passe ainsi d'une structure conforme à une structure non conforme, contrairement à CI-R dont la structure reste conforme.

L'anaphore du verbe (Creissels, 1993 : 76) ne se trouve pas dans le corpus. La pro-forme des verbes en français, le verbe FAIRE, n'est employée dans les données que dans le sens de FABRIQUER ou en tant que verbe-support dans FAIRE TOMBER (cf. 3.2.2).

L'analyse des pronoms dans des données comparables en anglais (enfants implantés vers 12 mois) montre un bon emploi de «it» / «this»comme sujet et comme complément d'objet direct, et de "them» comme complément d'objet direct et complément d'objet indirect. Nous observons par exemple les emplois de SPIT IT OUT vs SPIT OUT $+\mathrm{N}$ chez un enfant (CI-TD) de 43 mois (30 mois post-implant) :

\begin{tabular}{|l|l|l|}
\hline SPIT & Enfant CI-TD & Adulte en interaction avec CI-TD \\
\hline SPIT OUT SN & 0 & 2 \\
\hline SPIT SN OUT & 0 & 4 \\
\hline SPIT IT & 1 & 0 \\
\hline SPIT IT OUT & 6 & 7 \\
\hline
\end{tabular}




\begin{tabular}{|l|l|l|}
\hline SPIT OUT & 1 & 0 \\
\hline
\end{tabular}

SPIT n'est jamais employé avec un SN par l'enfant, alors que l'adulte emploie autant SPIT avec un SN (6 occurrences) qu'avec un pronom (7 occurrences); l'enfant emploie SPIT IT (1) / SPIT OUT (1) / SPIT IT OUT (6) comme une formule qui semble fonctionner en bloc avec un pronom (IT) passe-partout, peu chargé référentiellement.

Qu'observe-t-on en termes d'ordre d'apparition de ces différentes occurrences chez l'enfant? L'occurrence SPIT OUT (donc sans pronom) est la dernière à apparaître. Sur un seul type de verbe, il est difficile de tirer des conclusions aussi fortes que ce qui a été observé en français, mais on peut tout de même évoquer que la charge référentielle s'allège après plusieurs occurrences.

\section{Les causes possibles}

2 Quelles sont les causes possibles des fonctionnements observés? Ceux-ci peuvent être dus à la nature de l'opération de pronominalisation: le recours aux pronoms marque un mouvement vers l'implicite puisque le référent n'est alors accessible qu'indirectement, par co-référence. Cela suppose de maintenir le lien référentiel activé sur un empan variable. Or la gestion de l'implicite linguistique est apparemment une difficulté plus grande que celle de l'implicite situationnel, puisque dans les échanges recueillis pour l'étude préliminaire au projet DEL (Vincent-Durroux et al., 2018), nous constatons que l'implicite situationnel est fréquent, notamment dans des occurrences de taquinerie, d'humour, d'opposition ou de collaboration entre enfants et adultes. Cela pourrait expliquer qu'une étape de la langue enfantine consiste à devenir explicite, voire redondante, avant de pouvoir gérer l'implicite comme les adultes: «les enfants de 6 ans ont tendance à tout dire » (Martinot, $2005: 82$ ).

Certaines causes sont-elles propres à un type d'enfant?

Chez les enfants avec TSL, des difficultés dues à un déficit linguistique sont envisagées (Eyer \& Leonard, 1995, Gopnik \& Crago, 1991, Pizzioli \& Schelstraete, 2008) ainsi que des difficultés dues à une limitation des capacités de traitement (Bishop, 1992; Deevy \& Leonard, 2004 ; Montgomery, 2003) qui favoriseraient l'emploi de structures moins complexes et moins coûteuses en mémoire de travail.

75 Chez les enfants sourds avec implant cochléaire, la progression langagière semble suivre une trajectoire normale voire accélérée dans les premiers mois suivant l'implantation cochléaire avec un développement grammatical étroitement lié au développement du lexique dans les premiers stades (Le Normand, 2005 ; Nott, 2009a ; 2009b), mais les deux composantes se dissocient aux stades plus complexes du développement grammatical (Hallé et al,. 2015 : 275 ; Chilosi et al., 2013); dans ces stades plus complexes, on peut ranger la pronominalisation, complexe par la virtualisation et les mouvements syntaxiques (déplacement du clitique accusatif) qu'elle implique. Peut également être mise en cause la mémoire de travail chez les enfants implantés, avec des différences selon les langues : elle apparait comme moins bonne que chez leurs pairs entendants pour les petits sourds germanophones et anglophones, alors qu'elle est comparable chez les italianophones (Duchesne, 2016 : 125). La mémoire phonologique à court terme pourrait être plus particulièrement affectée par la surdité profonde, alors que les capacités de cette mémoire augmentent 
fortement chez les enfants entendants entre 2 et 9 ans (Baddeley, 2000), expliquant qu'on observe des omissions des pronoms anaphoriques accusatifs chez les enfants entendants seulement jusqu'à l'âge de 4 ans environ (Bourdin, 2015 : 472). La mémoire phonologique, au stade que nous étudions, pourrait suffire à l'appropriation par les enfants sourds de constructions d'amplitude limitée mais se révéler insuffisante pour des constructions plus longues telles que les structures transitives. Le fait d'entendre grâce à l'implant ne serait pas alors nécessaire et suffisant.

Pourrait-il y avoir des causes communes aux deux types d'enfants?

Du fait des points communs observés entre les deux groupes d'enfants, l'hypothèse d'une cause d'origine phonologique (les pronoms sont souvent peu accentués donc peu audibles) ne sort pas renforcée car les enfants avec TSL ne sont pas sourds. Cette hypothèse dite "phonologique " est soutenue par Svirsky et al. (2004) pour qui le développement morphologique dépend de la saillance perceptuelle des marqueurs grammaticaux; la perception via les implants n'est pas aussi riche et complexe que l'oreille (Hammer et al., 2014 ; Inscoe et al., 2009 ; Dubois-Bélanger et al., 2010) même si elle donne un meilleur accès aux fréquences élevées de la voix que les appareils auditifs numériques (Stelmachowicz et al., 2004); la représentation et l'organisation phonologique resteraient toutefois plus grossières chez les enfants implantés que chez les enfants entendants (Hallé et al., 2015 : 276), perturbant l'accès aux distinctions fines des phonèmes et donc des morphèmes.

La supériorité des résultats en compréhension sur ceux obtenus en production invite à faire l'hypothèse que ce n'est pas un déficit linguistique structurel qui est en cause, mais plutôt des facteurs de performance. Bourdin et al. (2016:164) envisage qu'il s'agisse d'une limitation des capacités cognitives, notamment la mémoire. Des déficits de la mémoire de travail ont été observés dans des tests impliquant la mémoire verbale immédiate, tels que la répétition de chiffres, de mots, de non-mots ou de phrases (Vincent-Durroux, 2014 ; Vieu et al., 2016; Sillon \& Vieu, 2012). Le développement grammatical pourrait être affecté par un déficit du mécanisme d'autorépétition subvocale chez les enfants sourds comme cela a été montré en cas de dysphasie (ou TSL) (Parisse \& Mollier, 2008) ainsi que dans des populations avec déficit intellectuel (Bussy et al., 2013).

La surcharge cognitive se révèle une cause possible et convaincante : chez les locuteurs experts, la plupart des traitements sont automatisés et entraînent peu de charge cognitive. Mais « le risque de surcharge cognitive est plus important dans les contextes où le langage ne se développe pas dans des conditions ordinaires" (Bourdin, 2015 : 471).

Plus qu'un critère linguistique, c'est donc un critère cognitif, susceptible de progression avec l'âge, qui pourrait être évoqué dans les productions spontanées que nous avons analysées. Le critère cognitif a également été retenu dans d'autres analyses que nous avons pu faire à propos d'autres formes telles que les prépositions, en rapport avec des représentations spécifiques chez les jeunes et les enfants sourds profonds relativement à l'espace et au temps (Vincent-Durroux, 2015). L'hypothèse cognitive pour expliquer les résultats décrits semble s'imposer au détriment de l'hypothèse phonologique / de perception. 


\section{Conclusion} que l'enfant a un développement typique. Cela laisse entrevoir qu'elle constituera une difficulté particulière pour l'enfant à développement atypique. Cet article a tenté de répondre aux questions posées dans l'introduction: quelles sont les étapes de l'acquisition des pronoms en L1 et quelles en sont les difficultés pour les enfants à développement atypique?

Plusieurs étapes sont nécessaires : la première étape est celle d'une bonne maîtrise de la structure syntaxique sujet-verbe-objet, avec des noms complets; peuvent ensuite se produire la virtualisation que représente la pronominalisation ainsi que les mouvements syntaxiques éventuels. Cet ordre est mis en évidence par le fait que l'emploi des clitiques objets demeure longtemps une difficulté pour l'enfant francophone, que son développement langagier soit typique ou atypique. Localiser les référents nommés, et marquer en syntaxe des référents sur lesquels s'établit le partage d'attention, est également une étape cruciale qui permet de mettre en place le volume phonologique des énoncés transitifs. Chez les enfants dont le développement n'est pas typique du fait de troubles divers, nos travaux révèlent en outre des difficultés dans la mise en place de la co-référence, notamment en contexte de référence suivie. La variation observée montre que l'acquisition des pronoms reste un défi de taille, en raison de multiples facteurs possibles, dont la charge cognitive qu'elle implique et les limitations relevées pour la mémoire de travail chez les enfants à développement atypique.

\section{BIBLIOGRAPHIE}

Aravind, A., Hackl, M. \& Wexler, K. (2017). Syntactic and Pragmatic Factors in Children's Comprehension of Cleft Constructions, Language Acquisition, 25(3), 284-314.

Baddeley, A.D. (2000). The episodic buffer: A new component of working memory? Trends in Cognitive Sciences, 4, 417-423.

Bain, R. (1936). The self-and-other words of a child. American Journal of Sociology, 41(6), 767-775.

Bishop, D.V. (1992). The underlying nature of specific language impairment. Journal of Child Psychology and Psychiatry, 33, 3-66.

Bourdin, B. (2015). Un modèle capacitaire du traitement langagier chez l'enfant sourd : le cas de la morphosyntaxe. ANAE 138, Approche neuropsychologique des apprentissages chez l'enfant, Surdité : évolutions technologiques, de la prise en charge et des apprentissages, 469-476.

Bourdin, B., Ibernon, L., Le Driant, B., Levrez, C. \& Vandromme, L. (2016). Troubles morphosyntaxiques chez l'enfant sourd et chez l'enfant dysphasique : similarités et spécificités. Revue de Neuropsychologie, Neurosciences cognitives et cliniques, 8(3), 161-172.

Corela, HS-35 | 2022 
Budwig, N. (1990 (rééd. 2014)). A functional approach to the acquisition of personal pronouns. In G. Conti-Ramsden \& C. Snow (eds.), Children's language, 7, 135-160. Hillsdale, New Jersey: Erlbaum. Budwig, N. (1995). A developmental-functionalist approach to child language. Hillsdale, New Jersey: Erlbaum.

Bussy, G., Rigard, C. \& Des Portes, V. (2013). Impact d'un entraînement de la mémoire à court terme verbale sur le langage d'enfants ayant une déficience intellectuelle. Revue francophone de la déficience intellectuelle, 24, 141-151.

Caët, S. (2013). Référence à soi et à l'interlocuteur chez des enfants francophones et anglophones et leurs parents. Thèse de doctorat en linguistique. Université Paris 3.

Caët, S. \& Morgenstern, A. (2015). First and second person pronouns in two mother-child dyads. In Gardelle, L. \& Sorlin, S. (eds.). The pragmatics of personal pronouns, 173-194. Amsterdam: John Benjamins Publishing Company.

Caselli, M. C., Rinaldi, P., Varuzza, C., Giuliani, A. \& Burdo, S. (2012). Cochlear implant in the second year of life: Lexical and grammatical outcomes. Journal of Speech, Language, and Hearing Research, 55(2), 382-394.

Chastain, C. (1975). Reference and context. In Gunderson, K. (ed.), Language mind and knowledge, 194-269.Minneapolis: University of Minnesota Press.

Childers, J. B. \& Tomasello, M. (2001). The role of pronouns in young children's acquisition of the English transitive construction. Developmental Psychology, 37(6), 739-748.

Chilosi, A.M., Comparini, A., Scusa, M.F., Orazini, L., Forli, F., Cipriani, P. \& Berrettini, S. (2013). A longitudinal study of lexical and grammatical development in deaf Italian children provided with cochlear implantation. Ear and Hearing, 34(3), e28-37.

Clark, E. V. (1978). From gesture to word: On the natural history of deixis in language acquisition. In Bruner, J. \& Garton, A. (eds.), Human growth and development: Wolfson College lectures 1976, 95-120. Oxford: Oxford University Press.

Clark, E. (1998). Lexique et syntaxe dans l'acquisition du français. Langue française, 118, 49-60.

Collombel-Leroy, M. \& Morgenstern, A. (2012). Rising grammatical awareness in a Frenchspeaking child from 18 months to 36 months: uses and misuses of possession markers. Journal of French Language Studies, 22, 57-75.

Cotte, P. (1996). L'explication grammaticale de textes anglais. Paris : Presses Universitaires de France. Cotte, P. (2008). Les propositions relatives et l'énonciation. Cycnos, 17. http://revel.unice.fr/ cycnos/index.html?id=1697

Creissels, D. (1993). Les notions primitives de la syntaxe. Réflexions sur l'articulation logique entre les notions sur lesquelles se fondent les descriptions grammaticales. In Maillard, M. (dir.), Lidil 8, Vers une rénovation de la grammaire et de sa terminologie, 51-83. Grenoble : Presses Universitaires de Grenoble.

Cronel-Ohayon, S. (2004). Etude longitudinale d'une population d'enfants francophones présentant un trouble spécifique du développement du langage : aspects syntaxiques. Thèse de doctorat. Sprimont, Université de Genève.

Deevy, P. \& Leonard, L.B. (2004). The comprehension of WH- questions in children with specific language impairment. Journal of Speech, Language, and Hearing Research, 47, 802-815. 
Deutsch, W. \& Budwig, N. (1983). Form and function in the development of possessives. Papers and Reports on Child Language Development, 22, 36-42.

Diessel, H. (2004). The acquisition of complex sentences. Cambridge: Cambridge University Press.

Dubois-Bélanger, R., Lavoie, M.-H., Duchesne, L. \& Bergeron, F. (2010). Morphosyntaxe réceptive d'enfants de 5 à 8 ans porteurs d'un implant cochléaire. Revue canadienne d'orthophonie et d'audiologie, 34(4), 271-281.

Duchesne, L. (2016). Grammatical Competence After Early Cochlear Implantation. In Marschark, M. \& Spencer, P. E. (eds.). The Oxford Handbook of Deaf Studies in Language, 113-131. Oxford: Oxford University Press.

Duchesne, L., Sutton, A. \& Bergeron, F. (2009). Language achievement in children who received cochlear implants between 1 and 2 years of age: Group trends and individual patterns. Journal of Deaf Studies and Deaf Education, 14(4), 465-485.

Fenson, L., Dale, P., Reznick, S., Thal, D., Bates, E., Hartung, J., Tethick, S. \& Reilly, J. (1993). MacArthur Communicative Development Inventories: User's guide and technical manual, San Diego, CA Singular Publishing Group.

Frank, M. C., Braginsky, M., Yurovsky, D. \& Marchman, V. A. (2017). Wordbank: An open repository for developmental vocabulary data. Journal of child language, 44(3), 677-694.

Eyer, J.A. \& Leonard, L.B. (1995). Functional categories and specific language impairment: A case study. Language Acquisition, 4, 177-203.

Girouard, P. C., Ricard, M. \& Gouin Décarie, T. (1997). The acquisition of personal pronouns in French-speaking and English-speaking children. Journal of Child Language, 24(2), 311-326.

Gopnik, M. \& Crago, M (1991). Familial aggregation of a developmental language disorder. Cognition, 39, 1-50.

Guasti, M. T., Papagno, C., Vernice, M., Ceccheto, C., Giuliani, A. \& Burdo, S. (2012). The effect of language structure on linguistic strengths and weaknesses in children with cochlear implants: Evidence from Italian. Applied Psycholinguistics, 5(4), 739-764.

Gundel, J. K., \& Johnson, K. (2013). Children's use of referring expressions in spontaneous discourse: Implications for theory of mind development. Journal of Pragmatics, 56, 43-57. DOI: 10.1016/j.pragma.2013.04.003

Hallé, F. \& Duchesne, L. (2015). Habiletés morpho-syntaxiques des enfants sourds porteurs d'implants cochléaires : une revue systématique. Revue canadienne d'orthophonie et d'audiologie, 39(3), 260-297.

Hammer, A., Coene, M., Rooryck, J. \& Govaerts, P. J. (2014). The production of Dutch finite verb morphology: A comparison between hearing-impaired CI children and specific language impaired children. Lingua, 139, 68-79.

Inscoe, J. R., Odell, A., Archbold, S. \& Nikolopoulos, T. P. (2009). Expressive spoken language development in deaf children with cochlear implants who are beginning formal education. Deafness and Education International, 11(1), 39-55.

Jacq, G., Tuller, L. \& Fuet, F. (1999). Spécificités morpho-syntaxiques du français de l'enfant sourd : une étude comparative. Glossa, 69, 4-14.

Jakubowicz, C. \& Rigaut, C. (2000). L'acquisition des clitiques nominatifs et des clitiques objets en français. Revue canadienne de linguistique, 45, 119-157. 
Jørgensen, R.N., Dale, P. S., Bleses, D. \& Fenson L. (2010). CLEX: A cross-linguistic lexical norms database. Journal of Child Language, 37(2), 419-428.

Kern, S. \& Gayraud, F. (2010). L'inventaire français du développement communicatif, Grenoble, La Cigale.

Kirjavainen, M., Theakston A., \& Lieven E. (2009). Can input explain children's me-for-I errors? Journal of child language 36 (5), 1091-1114.

Le Normand, M.-T. (2005). Production du lexique chez des enfants sourds profonds munis d'un implant cochléaire sur un suivi de quatre ans. Handicap, Revue de sciences humaines et sociales, 105-106, 21-31.

Leonard, L.B. (1998). Children with specific language impairment. Cambridge: The MIT Press.

Lieven, E. (2014). First language development: a usage-based perspective on past and current research. Journal of Child Language, 41, 48-63.

Loveland, K. A. (1984). Learning about points of view: spatial perspective and the acquisition of 'I/ you'. Journal of Child Language, 11(3), 535-556.

Martel, K. \& Dodane, C. (2012). Le rôle de la prosodie dans les premières constructions grammaticales : étude de cas d'un enfant français monolingue. French Language Studies, 22, 13-35.

Martinot, C. (2005). Comment parlent les enfants de 6 ans? Pour une linguistique de l'acquisition. Besançon : Presses universitaires de Franche-Comté.

Montgomery, J. (2003). Working memory and comprehension in children with specific language impairment: what we know so far. Journal of Communication Disorders, 36, 221-231.

Morgenstern, A. (1995). L'enfant apprenti-énonciateur. (L'auto-désignation chez l'enfant en français, en anglais et en Langue des Signes Française). Thèse de doctorat. Université Paris 3.

Morgenstern, A. (2003). Le langage de l'enfant est-il linguistiquement correct ? In Delmas, C. (dir.), Correct, incorrect en linguistique anglaise, CIEREC Travaux 113, 111-128. Saint- Etienne : Publications de l'Université de Saint-Etienne.

Morgenstern, A. (2006). Un JE en construction. Ontogénèse de l'autodésignation chez l'enfant. Paris et Gap : Ophrys.

Morgenstern, A. (2012). The self as other: Self words and pronominal reversals in language acquisition. In Lorda, C. U. \& Zabalbeascoa, P. (eds.), Spaces of polyphony, 57-72. Amsterdam: John Benjamins Publishing Company.

Morgenstern, A. \& Parisse, C. (2007). Codage et interprétation du langage spontané d'enfants de 1 à 3 ans. Corpus, interprétation, contextes, codage, 6, 55-78.

Müller, N. \& Kupisch, T. (2007). Acquisition des déterminants et des clitiques objets chez des enfants bilingues (français-allemand). Acquisition et interaction en langue étrangère, (25), 45-68.

Naigles, L. \& Hoff-Ginsberg, E. (1998). Why are some verbs learned before other verbs? Effects of input frequency and structure on children's early verb use, Journal of Child Language, 25, 95-120.

Nott, P., Cowan, R., Brown, P.M \& Wigglesworth, G. (2009a). Early language development in children with profound hearing loss fitted with a device at a young age: Part I - the time period taken to acquire first words and first word combinations. Ear and Hearing, 30(5), 526-540.

Nott, P., Cowan, R., Brown, P.M \& Wigglesworth, G. (2009b). Early language development in children with profound hearing loss fitted with a device at a young age: Part II - content of the first lexicon. Ear and Hearing, 30(5), 541-551. 
Paradis, J., Crago, M. \& Genesee, F. (2002). Object clitics as a clinical marker of SLI: evidence from French bilingual children. Proceedings of the $27^{\text {th }}$ Boston University conference on Language Development.

Parisse, C. \& Maillart, C. (2004). Le développement morpho-syntaxique des enfants présentant des troubles du développement du langage : données francophones. Enfance, 56, 20-35.

Parisse, C. \& Mollier, R. (2008). Le déficit de mémoire de travail chez les enfants dysphasiques estil ou non spécifique du langage ? In Durand, J., Habert, B. \& Laks, B. (eds). Congrès mondial de linguistique française. Paris : Institut de linguistique française.

Pizzioli, F. \& Schelstraete, M. (2008). The argument-structure complexity effect in children with specific language impairment: evidence from the use of grammatical morphemes in French. Journal of Speech, Language, and Hearing Research, 51, 706-721.

Qi, R. (2005). From nominal to pronominal person reference in the early language of a MandarinEnglish bilingual child. In ISB4: Proceedings of the 4th International Symposium on Bilingualism, 18931909.

Rossi, C. (2017). Mouvement et développement du langage. In Morgenstern, A. \& Parisse, C. (dir.). Le langage de l'enfant : de l'éclosion à l'explosion, 100-117. Paris : Presses Universitaires de la Sorbonne Nouvelle.

Rowland, C. F. (2007). Explaining errors in children's questions. Cognition, 104(1), 106-134.

Sabeau-Jouannet, E. (1975). Les premières acquisitions syntaxiques chez des enfants français unilingues. La linguistique, 11(1), 105-122.

Salazar Orvig, A., Marcos, H., Morgenstern, A., Hassan, R., Leber-Marin, J. \& Parès, J. (2010). Dialogical factors in toddlers' use of clitic pronouns. First Language, 30(3-4), 374-402.

Salazar Orvig, A., \& Morgenstern, A. (2015). Acquisition and use of pronouns in a dialogic perspective. The Acquisition of Reference. Trends in Language Acquisition Research, 155-180.

Schnedecker, C. \& Landragin, F. (2014). « Les chaînes de référence : présentation », Langages 195, 3-22. Paris : Larousse/Armand Colin.

Sekali, M. (2012). The emergence of complex sentences in a French child's language from 0;10 to 4;01: causal adverbial clauses and the concertina effect. Journal of French Language Studies, 22 (1), 115-141.

SFORL (Société française d'ORL et de chirurgie de la face et du cou) (2018). Recommandation pour la pratique clinique. Indications de l'implant cochléaire chez l'adulte et chez l'enfant.

https://www.orlfrance.org/wp-content/uploads/2018/09/

RCP_SFORL_Indications_implant_cochleaire_adulte_enfant_2018.pdf

Sinclair-de-Zwart, H. (1969). Developmental psycholinguistics. In Elkind, D. \& Flavell, J. H. (eds.), Studies in cognitive development: essays in honor of Jean Piaget, 315-336. New York: Oxford University Press.

Slobin, D. I. (1985). Cross linguistic evidence for the Language-Making Capacity. In Slobin, D. I. (ed.), The crosslinguistic study of language acquisition. Vol. 2: Theoretical issues, 1157-1256. Hillsdale, NJ: Lawrence Erlbaum Associates.

Stelmachowicz, P. G., Pittman, A. L., Hoover, B. M., Lewis, D. E. \& Moeller, M. P. (2004). The importance of high-frequency audibility in the speech and language development of children with hearing loss. Archives of Otolaryngology-Head and Neck Surgery, 130(5), 556-562. 
Svirsky, M.A., Teoh, S. W. \& Neuburger, H. (2004). Development of Language and Speech Perception in congenitally, profoundly deaf children as a function of age at cochlear implantation. Audiology and Neurotology, 9(4), 224-233.

Thordardottir, E. T. (2005). Early lexical and syntactic development in Quebec French and English: implications for cross-linguistic and bilingual assessment. International Journal of Language \& Communication Disorders, 40(3), 243-278.

Tomasello, M. (1998). One child's early talk about possession. In Newman, J. (ed.) The Linguistics of Giving, 349-374. Amsterdam: John Benjamins Publishing Company.

Tomblin, J.B., Barker, B.A., Spencer, L. J., Zhang, X. \& Gantz, B.J. (2005). The effect of age at cochlear implant initial stimulation on expressive language growth in infants and toddlers. Journal of Speech, Language, and Hearing Research, 48(4), 853-867.

Tuller, L. \& Jakubowicz, C. (2004). Développement de la morphosyntaxe du français chez des enfants sourds moyens. Le langage et l'homme, 39, 191-208.

Sillon, M. \& Vieu, A. (2012). La lecture indirecte minutée : un outil pour l'évaluation des compétences auditives. Rééducation orthophonique, 252, 109-125.

Vieu, A., Yoka, A., Sillon, M., Guiheneuf, A., Durroux, L., Artières Sterkers F., Mondain, M. \& Uziel, A. (2016). Compréhension de l'implicite chez de jeunes adultes implantés précocement. Rééducation orthophonique, 268, 203-220.

Vincent-Durroux, L. (2014). La langue orale des jeunes sourds profonds. Bruxelles : Editions de BoeckSolal, collection Voix, Parole, Langage.

Vincent-Durroux, L. (2015). L'expression de l'aspect chez les locuteurs sourds : quel rôle pour les prépositions ? In Merle, J.-M. (dir.), Prépositions et aspectualité, Faits de Langues, 69-77. Berne :

Peter Lang.

Vincent-Durroux, L., Martel, K., Guiheneuf, A. \& Vieu, A. (2018). Les structures verbales chez deux jeunes enfants francophones sourds profonds avec implant cochléaire. Emergence et développement à 36 mois post-implant. Language, Interaction and Acquisition (LIA), 9(2), 229-258.

https://doi.org/10.1075/lia.17007.vin

\section{NOTES}

1. Le site CLEX, qui est plus ancien, fonctionne toujours, et on y trouve notamment la version américaine des inventaires, ainsi que beaucoup d'autres données librement accessibles depuis ce site, cf. « CLEX : A cross-linguistic lexical norms database » (Jørgensen et al., 2010)

2. C'est la base Wordbank, qui peut être interrogée à l'adresse suivante: http:// wordbank.stanford.edu/ (dernière consultation le 11 juillet 2018).

3. La consigne donnée aux parents dans les inventaires précise bien que les formes considérées comme produites doivent correspondre à des productions spontanées de l'enfant (par opposition à des répétitions directes du discours adressé ou entendu).

4. Nous avons intégré les adverbes qui font l'objet des mêmes usages déictiques que les pronoms.

5. la phrase est inachevée, elle marque l'exaspération.

6. Le corpus est consultable et téléchargeable depuis le site du projet: http://colaje.scicog.fr/ index.php/corpus

7. Ce suivi longitudinal fait également partie du corpus CoLaJe (cf. supra) 
8. Les données ont été recueillies à l'Institut Saint-Pierre (Palavas) et à The Elizabeth Foundation (Cosham, Portsmouth) dans le cadre de partenariats de recherche. Nous remercions les participants à cette étude.

\section{RÉSUMÉS}

L'acquisition des pronoms chez les enfants à développement langagier typique est complexe car elle combine de nombreux paramètres, notamment une capacité d'abstraction. Dans une première partie, ces paramètres sont décrits en rapport avec les trajectoires d'apprentissage mises en évidence par la littérature (par des tests et dans des données spontanées). La seconde partie est consacrée aux difficultés que pose l'acquisition des pronoms chez des enfants à développement langagier atypique, pour des raisons de troubles spécifiques du langage ou de surdité profonde.

The acquisition of pronouns in typically developing children is complex because it combines many parameters, including the ability to abstract. In the first part, these parameters are described in relation to the learning trajectories highlighted in the literature (from tests and in spontaneous data). The second part is devoted to the difficulties posed by the acquisition of pronouns in children with atypical language development, due to specific language disorders or profound deafness.

\section{INDEX}

Mots-clés : acquisition, pronom, développement typique du langage, développement atypique du langage, TSL, surdité

Keywords : acquisition, pronoun, typical language development, atypical language development, specific language disorders, deafness

\section{AUTEURS}

\section{CAROLINE ROSSI}

Université Grenoble Alpes - ILCEA4

\section{LAURENCE VINCENT-DURROUX}

Université Grenoble Alpes - LIDILEM 